
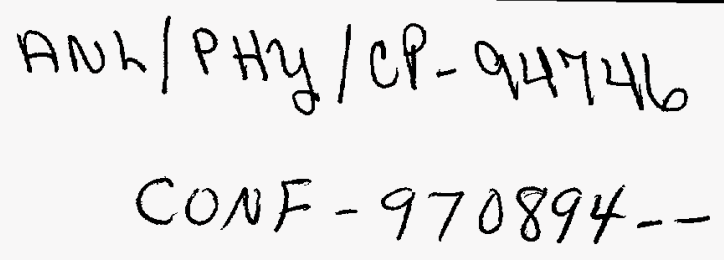

\title{
Performance of the Laser Driven Polarized Hydrogen Source at IUCF
}

\section{R. V. Cadman ${ }^{1 a}$, K. Bailey ${ }^{b}$, J. Brack $^{c}$, W. J. Cummings ${ }^{b}$, J. Fedchak ${ }^{b}$, B. Fox ${ }^{c}$, H. Gao ${ }^{2 b}$, C. Grosshauser ${ }^{d}$, R. J. Holt ${ }^{a}$, C. E. Jones ${ }^{e}$, E. Kinney ${ }^{c}$, W. Kirwin ${ }^{c}$, R. S. Kowalczyk ${ }^{b}$, Z.-T. Lu ${ }^{b}$, M. A. Miller ${ }^{a}$, W. Nagengast ${ }^{d}$, B. R. Owen ${ }^{a}$, K. Rith ${ }^{d}$, F. Schmidt ${ }^{d}$, E. C. Schulte ${ }^{a}$, J. Sowinski ${ }^{f}$,

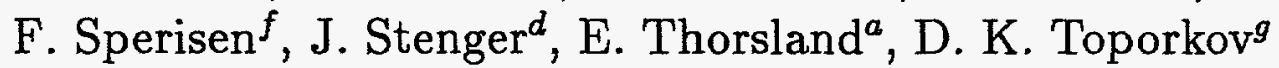

a University of Illinois, Urbana, Illinois 61801

${ }^{b}$ Physics Division, Argonne National Laboratory, Argonne, Illinois 60439

${ }^{c}$ University of Colorado, Boulder, Colorado 80309-0446

${ }^{d}$ Universität Erlangen-Nürnberg, 91058 Erlangen, Germany

${ }^{e}$ California Institute of Technology, Pasadena, California 91125

${ }^{f}$ Indiana University Cyclotron Facility, Bloomington, Indiana 47408

${ }^{9}$ Budker Institute for Nuclear Physics, 630090 Novosibirsk, Russia
RECEIVED

NOV 041997

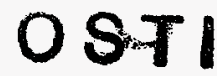

\begin{abstract}
A laser driven source of polarized hydrogen and deuterium has been installed in the Cooler Ring at the Indiana University Cyclotron Facility. Polarized nuclei from the source are injected into a storage tube, and the resulting target has been used in a scattering experiment with $200 \mathrm{MeV}$ proton beam. This paper discusses the performance of the source, including measurements of atomic fraction and electron polarization.
\end{abstract}

For several years there has been interest in using spin exchange with an optically pumped polarized alkali vapor as a way of obtaining a source of highly polarized hydrogen or deuterium nuclei with a higher flux than can be achieved by other methods [1]. Development of this technique on the bench led to a target which, assuming spin-temperature equilibrium, had a higher figure of merit than conventional targets currently in use [2]. The source was then installed in the Cooler Ring at the Indiana University Cyclotron Facility (IUCF) and has now been tested with the proton beam. The analysis of

1) Corresponding author, cadman@uiuc.edu

2) Present address: Laboratory for Nuclear Science, Massachusetts Institute of Technology, Cambridge, Massachusetts 02139 


\section{DISCLAIMER}

This report was prepared as an account of work sponsored by an agency of the United States Government. Neither the United States Government nor any agency thereof, nor any of their employees, makes any warranty, express or implied, or assumes any legal liability or responsibility for the accuracy, completeness, or usefulness of any information, apparatus, product, or process disclosed, or represents that its use would not infringe privately owned rights. Reference herein to any specific commercial product, process, or service by trade name, trademark, manufacturer, or otherwise does not necessarily constitute or imply its endorsement, recommendation, or favoring by the United States Government or any agency thereof. The views and opinions of authors expressed herein do not necessarily state or reflect those of the United States Government or any agency thereof. 


\section{DISCIAMER}

Portions of this docament may be illegible in electronic image products. Images are produced from the best avanlable origion docoment. 
nuclear polarization data is not complete and is discussed in the paper by $M$. Miller in this volume. Here we present data on the performance of the source in terms of electron polarization and atomic fraction.

The hydrogen nuclei are polarized in the glassware shown in figure 1. Molecules enter from the right and are dissociated by an rf plasma discharge. The atoms then enter the spin exchange cell. At the left a reservoir of potassium is heated and the vapor flows into the spin exchange cell. Circularly polarized light from a Ti: $\mathrm{Al}_{2} \mathrm{O}_{3}$ laser enters the spin exchange cell from the top. Its polarization is transferred to the hydrogen nuclei in three steps: first to the potassium valence electrons through photoabsorption, then to the hydrogen electrons through $\mathrm{K}-\mathrm{H}$ spin exchange collisions, and finally to the hydrogen nuclei through $\mathrm{H}-\mathrm{H}$ spin exchange collisions. The polarized hydrogen exits the spin exchange cell and enters an aluminum storage tube through which the proton beam passes. Some atoms pass directly from the spin exchange cell through a hole in the bottom of the storage tube and into the atomic polarimeter [1] which was used for the measurements reported in this paper.

The source used at IUCF differs from other laser driven sources in two ways. First, we have eliminated the transport tube, which decreases the number of wall bounces and thus improves the atomic fraction and polarization. Second, we no longer use a uniform magnetic field. In a typical magnet setting the field in the lower half of the spin exchange cell will vary between $110 \mathrm{mT}$ and 120 $\mathrm{mT}$, but decreases to about $20 \mathrm{mT}$ at the top of the cell. This leads to a region near the bottom of the cell where potassium can be optically pumped without radiation trapping, and a region at the top where polarization is transferred from hydrogen electrons to hydrogen nuclei more rapidly.

Figure 2 shows the performance of the source during a test run of the target. These data were taken with a flow of $1 \times 10^{18}$ atoms/sec, with a 6 $\mathrm{mm}$ diameter hole between the spin exchange cell and the storage tube, and with $1.5 \mathrm{~W}$ of laser power at the source. The figure shows that increasing potassium temperature led to an increase in the polarization and a decrease in the atomic fraction. Eventually the high potassium density in the spin exchange cell caused nearly complete molecular recombination.

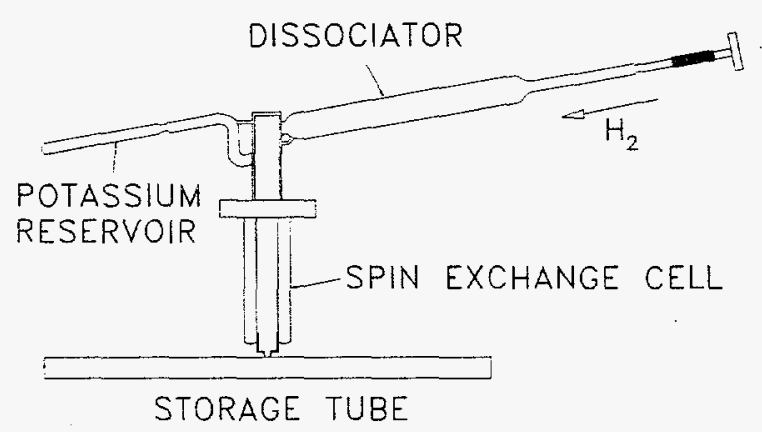

FIGURE 1. Glassware used for the Laser Driven Source 

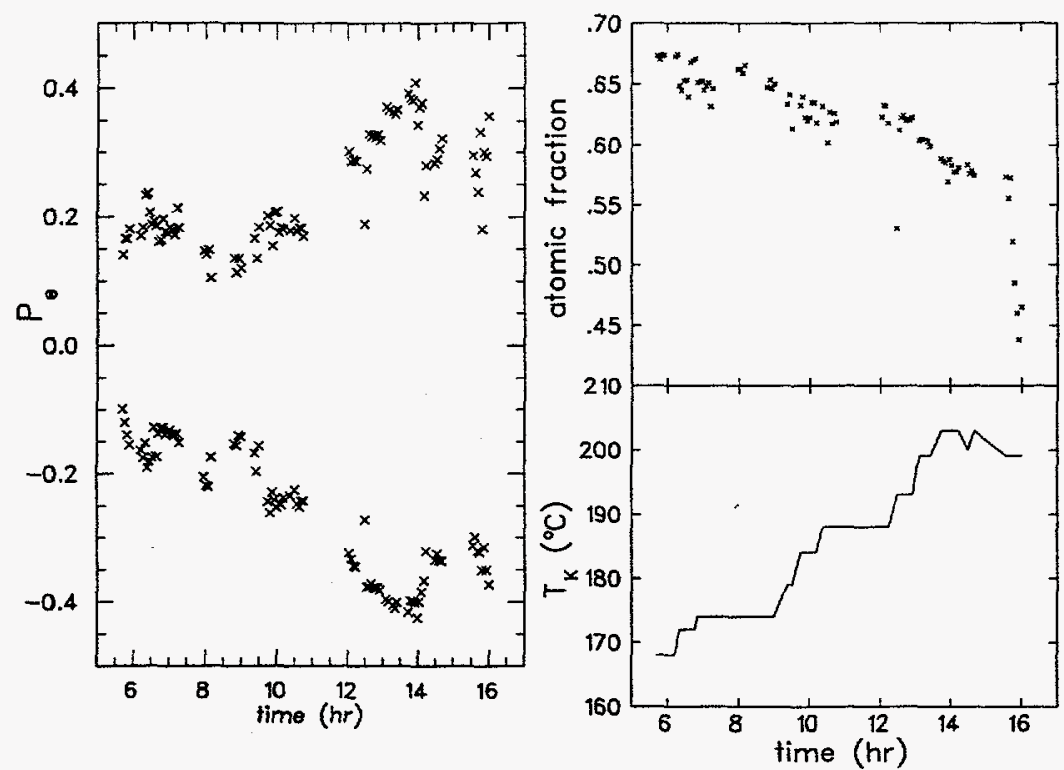

FIGURE 2. Performance of the hydrogen source during tests with the proton beam. Clockwise from left: electron polarization; atomic fraction; and temperature of the potassium reservoir over a 10 hour period.

More recently tests were made without the proton beam in preparation for future beam time. A $4 \mathrm{~mm}$ hole was used between the spin exchange cell and the storage tube, and the glass was coated using the afterwash technique described in ref. [3]. As expected from the smaller hole size, we found a lower atomic fraction, about $40 \%$. We also found higher polarizations, and observed that doubling our laser power by adding a second laser made a significant improvement. The best result was a polarization of $63 \%$ with an atomic fraction of $35 \%$, again at $1 \times 10^{18}$ atoms $/ \mathrm{sec}$.

We thank D. Tupa for the loan of the two Ti: $\mathrm{Al}_{2} \mathrm{O}_{3}$ lasers, our glass blower W. Lawrence, A. Kenyon for helping with assembly and repair of the source, J. Doskow for maintaining our vacuum system, and the rest of the operators and staff at IUCF for all their assistance. This work was supported in part by the NSF under grants PHY94-20787 and PHY94-20470 and by the DOE under contract No. W-31-109-ENG-38 and grant DE-FG02-86ER40269.

\section{REFERENCES}

1. Poelker, M., et al., Phys. Rev. A 50, 2450 (1994).

2. Owen, B., et al., in Proceedings of the 12th International Symposium on HighEnergy Spin Physics, Amsterdam, 1996, edited by C. W. de Jager, et al. (World Scientific, Singapore, 1997), p. 490.

3. Fedchak, J. A., et al., Nucl. Instrum. Methods Phys. Res., Sect. A 391, 405 (1997). 